\title{
La arquitectura del silencio: lo vernáculo en las viviendas del Valle del Chota (1960-1990)
}

\section{The architecture of silence: the vernacular in the dwellings of Valle del Chota (1960-1990)}

Naranjo Toro Miguel Edmundo

Universidad Técnica del Norte, Ecuador

Leyva Yenney Ricardo

Universidad Técnica del Norte, Ecuador

Cruz Hernández Diana María

Universidad Técnica del Norte, Ecuador

Autor para correspondencia: yenneyrl@ hotmail.com

Fecha de recepción: 15 de diciembre de 2017 - Fecha de aceptación: 26 de febrero 2018

Resumen: El objetivo principal de esta investigación radica en establecer las principales características de la vivienda vernácula en el Valle del Chota. Este estudio se ha desarrollado con una visión holística desde la perspectiva de la metodología cualitativa y del paradigma hermenéutico. Se trata de un estudio de caso, por lo que los métodos capitales fueron la observación científica documental y el trabajo de campo. La principal interrogante que rigió el estudio fue: ¿cuáles son los valores patrimoniales de la vivienda vernácula en el Valle del Chota a partir del influjo cultural afrodescendiente, y cómo éstos se relacionan con la vida cotidiana y la cultura material y espiritual de la región dentro de las aspiraciones actuales del Sumak Kawsay o Buen vivir? Se trata de una arquitectura que dialoga con el entorno y mantiene ese originario principio de intercambio entre hombre y medio ambiente, a través de la vivienda.

Palabras claves: arquitectura; vernácula; identidad; patrimonio; vivienda; Valle del Chota

Abstract: The main objective of this research is to establish the main features of vernacular housing in the Chota Valley. This study has been developed with a holistic perspective of qualitative methodology and the hermeneutic paradigm. It is a case study, so the capital methods were the observation scientific documentary and field work. The main question which governed the study was: what the heritage values of the vernacular dwelling in the Chota Valley from the cultural influence of African descent, and how these relate to everyday life and material culture and Spiritual of the region within the current aspirations of the Sumak Kawsay or good live? It's an architecture with the environment and maintains the original principle of exchange between man and environment, trough housing.

Key words: architecture; vernacular; identity; heritage; living place 


\section{Introducción}

El Valle del Chota está situado en la Sierra Norte del Ecuador -provincias de Imbabura y Carchi-; allí se han desarrollado asentamientos con estructuras habitables de filiación vernácula. Se trata de una arquitectura que dialoga con el entorno y mantiene ese originario principio de intercambio entre hombre y medio ambiente, a través de la vivienda. Por la singularidad tipológica que ella aporta debe ser valorada como una parte significativa del patrimonio cultural tangible ecuatoriano. Este modo de edificar se distingue por el uso de materiales locales como el adobe, la piedra, la madera y las tejas de barro en la concepción de estructuras sencillas y funcionales para la habitación de una población, fundamentalmente afro ecuatoriana dedicada a la agricultura que conserva expresiones culturales ancestrales. Estas construcciones, han padecido la invisibilidad, tal vez, porque se encuentran sin duda muy alejadas de los modelos formales europeos.

El objetivo principal que motivó el desarrollo esta investigación fue establecer las principales características de la vivienda vernácula en el Valle del Chota en el período 19601990. La cardinal interrogante que rigió el estudio fue: ¿cuáles son los valores patrimoniales de la vivienda vernácula en el Valle del Chota a partir del influjo cultural afro descendiente, y cómo éstos se relacionan con la vida cotidiana y la cultura material y espiritual de la región dentro de las aspiraciones actuales del Sumak Kawsay o Buen vivir? La investigación constituye un aporte a la historia de la arquitectura del Ecuador. Las casas objeto de análisis, devienen edificaciones y lugares que expresan la mentalidad colectiva, los imaginarios sociales y reproducen discursos simbólicos de sus habitantes. Se pretende es poner en valor la vivienda afro ecuatoriana como reservorio de prácticas ancestrales en tanto elemento distintivo del patrimonio histórico y cultural del Valle del Chota, y fehaciente vehículo identidad. Por ello, la hipótesis defendida se sustenta en la idea de que la vivienda vernácula en el Valle del Chota constituye parte del patrimonio construido y tiene valores socioculturales, funcionales, simbólicos, estéticos, históricos y espirituales, plasmando un proceso continuo que incluye cambios adaptativos en correspondencia con las transformaciones económicas y sociales que definen la identidad la comunidad.

Se está convencido de que puesta en valor no es un término sustituto de conservación, protección, restauración; sin embargo, se considera que para gestionar y proteger primero hay que conocer e investigar a profundidad y críticamente el objeto de actuación. Entonces, se aboga por que las investigaciones científicas y sus autores no queden al margen de la instrumentalización de políticas conservacionistas; pues para lograr una eficaz protección y preservación del patrimonio, ambos procesos deben funcionar o actuar de forma coordinada y consensuada.

La puesta en valor de la vivienda vernácula contribuiría a reforzar la relevancia social e histórica de las comunidades locales negras campesinas, verdaderas protagonistas de la construcción del entorno de una parte del medio rural ecuatoriano. Además, este marco de partida sienta las bases para una adecuada discusión que considere la historicidad de los procesos políticos, sociales y económicos que, en cada momento, mediaron en la cotidianeidad de las personas que transformaron su entorno y dieron significados culturales a través de su propia identidad a este contexto hoy considerados ancestrales. 
En la revisión documental realizada se pueden destacar como textos medulares: Cobijo y Sociedad de Paul Oliver(1969) ${ }^{1}$ y en el contexto latinoamericano el trabajo Arquitectura vernácula, fundamento en la enseñanza de sustentabilidad, de Torres Zárate (2014). En el Ecuador, fue esencial el aporte de estudios como Coangue o Historia Cultural y Social de los negros del Chota y Salinas, de Piedad Peñaherrera y Alfredo Costales (1959), a pesar de su fecha de publicación ofrece una valoración específica acerca de la vivienda vernácula del Valle del Chota, por lo que pudiera ser considerado como un antecedente referencial obligado. Otros antecedentes, importantes lo constituyen Arquitectura vernácula en el litoral por David Nurnberg (1882) y Arquitectura vernácula - Arquitectura con arquitectos: una interacción fecunda en riesgo de extinción (Cultura urbana), de Enrique Ortiz (1999). Destrucción de la arquitectura vernácula campesina en los Andes ecuatorianos, de Arrata (2014); entrega significativas referencias a la desaparición paulatina de esta arquitectura considerada auténtica por sus valores intrínsecos. Del mismo modo, Análisis de la arquitectura vernácula del Ecuador: Propuestas de una arquitectura contemporánea sustentable, de Yépez (2012), particulariza y define los rasgos distintivos de esta tipología en cada una de las regiones del país: Costa, Sierra, Amazonía e Insular. Saravino (2011). Por su parte, Arquitectura indígena: fundamentos para la generación de una arquitectura contemporánea, pondera los valores de los procedimientos originarios como vía para solucionar problemáticas actuales tanto en el país como en otros ámbitos ${ }^{2}$. Algunos autores consideran que la arquitectura vernácula es resultado de un lento proceso histórico en el cual se mezclan elementos indígenas, africanos y europeos ${ }^{3}$. Esta, aprovecha los materiales a los que se tiene acceso en cada región y su único objetivo es ofrecer cobijo, sin otras pretensiones ${ }^{4}$.

En el marco de esta pesquisa se entiende como vivienda vernácula todas aquellas edificaciones que de manera empírica diseña y construye el ser humano, sin mayor inversión económica a fin de dotarse a sí mismo y a su comunidad de vivienda y "todas" las comodidades anexas para la satisfacción de sus necesidades (vivienda, cultura, relaciones sociales y de producción), para lo cual utiliza materiales del contexto en que fabrica sin deteriorar el ambiente (tierra, piedra, madera y sus derivados) y que deviene espacio esencial para el despliegue de sus manifestaciones culturales tradicionales.

De igual forma, otros textos apuntalaron el análisis, como por ejemplo los artículos La categoría de "afroecuatoriano" y los rasgos de autoidentificación étnica en censos y encuestas de Ecuador de John Antón (2008) y de Iván Pabón (2006) Procesos de construcción identitaria en las comunidades negras de la cuenca Chota-Mira en tres generaciones: abuelos, adultos mayores y jóvenes.El autor centra su explicación en las peculiaridades de la historia de los africanos al

\footnotetext{
${ }^{1}$ Carta del Patrimonio Vernáculo Construido de 1999, (ICOMOS, 1999).

${ }^{2}$ Sobre el mismo contexto ecuatoriano se consultaron además los trabajos: Saraguros mantienen sus viviendas tradicionales (2015) y La arquitectura vernácula en el medio rural y urbano de Manabí, de Sandoval, Machuca \& Cedeño (2014), los cuales permiten encontrar aristas comunes y diferenciadas de las expresiones de su arquitectura vernácula.

${ }^{3}$ Se han considerado legislaciones emanadas de: el Centro Internacional de Monumentos y Sitios (ICOMOS), el Consejo Internacional de Museos (ICOM), el Centro Internacional de Estudios para la Conservación y la Restauración de Bienes Culturales (ICCROM), la Unión Internacional de Arquitectos (UIA), el Centro del Patrimonio Mundial y el Consejo de Europa y la Organización de las Naciones Unidas para la Educación, la Ciencia y la Cultura (UNESCO).

${ }^{4}$ Para el estudio de la arquitectura vernácula ecuatoriana hay que tener en cuenta que existen cuatro regiones: la costa, la sierra, la Amazonía y la insular. Cada una de ellas amerita un estudio independiente porque presentan rasgos que las identifican y diferencian a una de otra de acuerdo con el contexto.
} 
Valle a través de un estudio de larga duración. En esta misma cuerda se ubica el texto Etnicidad, Actores y Territorio: Luchas de Los Afrodescendientes de Patía y El Valle del Chota por el acceso a tierra y agua, 19902010, de Diana Patricia Angulo Balanta(2013). La autora, se concentra en analizar las estructuras organizativas, luchas sociales y redefiniciones identitarias de las comunidades y pueblos afrodescendientes del Patía en Colombia y el Valle del Chota en Ecuador, referidas a las demandas de acceso a tierra y agua, desde una perspectiva de análisis comparado. A esta perspectiva se suma, Esclavos de La Tierra: Los Campesinos Negros del Chota-Mira, Siglos XVII-XX( 1997) de Ernrnanuelle Bouisson y Miradas del Pueblo Afroecuatoriano hacia la Construcción de una Agenda Inclusiva para alcanzar la Justicia, su Reconocimiento y el (Ubuntu) Desarrollo (2016), elaborado por la Secretaría Nacional de Gestión de la Política y el Consejo de Participación y Control Social con la intención de dar seguimiento a los objetivos, metas y acciones contempladas en el Decenio afro. El texto Estrategias para el fortalecimiento del turismo cultural en las comunidades del Juncal, Chalguayacu y Carpuela provincia de Imbabura ${ }^{5}(2015)$ de Omayda Padilla Delgado, se propone como objetivo general diseñar estrategias de fortalecimiento para el turismo cultural en las comunidades del Juncal, Chalguayacu y Carpuela, a partir del diagnóstico de la situación actual del turismo cultural de las comunidades. Si bien resulta una alentadora propuesta la autora no muestra en concordancia con las narrativas oficiales lanzadas por las instituciones y organismos públicos encargados de la gestión y promoción del Turismo y el Patrimonio natural y cultural choteño alrededor del significante "Valle del Chota Territorio Ancestral", no se muestra crítica hacia las implicaciones sociológicas o las consecuencias de estas narrativas públicas a nivel práctico para los habitantes del medio rural. Las campañas de promoción turística erigidas desde los discursos oficiales alrededor del lema «Valle del Chota territorio Ancestral» dejan en sombras la marginación de las poblaciones afroecuatrianas hecho que se suma a la creciente subordinación de las comunidades locales en los procesos de deliberación y toma de decisiones acerca de la gestión de sus propios territorios.

Como se había señalado, algunos estudios se dedican a la temática de los valores patrimoniales en el Valle del Chota, en particular en relación con el turismo. Ello, ha dado lugar a ensayos, investigaciones y foros académicos en el ámbito cultural; que alumbran el escenario literario sobre el tema. Los que aquí se mencionan, en tanto antecedentes decisivos, para la concepción de esta investigación, señalan no solo aspectos que definen la presencia del interés actual por este tema como tal, sino que además exponen herramientas válidas para la observación de las funciones que dichos aspectos pueden tener, a partir de lo cual se hace posible, también, marcar diferencias entre las posturas sugeridas. Las fuentes son de diversa naturaleza, documentales, artículos de publicaciones periódicas, monografías, actas de congresos cartas, documentos técnicos de algunas instituciones y una amplia gama de documentos on line y páginas web de organizaciones importantes.

El análisis de los antecedentes en la investigación, relativos al Valle del Chota, Chalguayacu y la relación existente entre patrimonio cultural, implica un enorme esfuerzo de síntesis y delimitación temática necesarios para contextualizar el objeto de estudio. Como se

\footnotetext{
${ }^{5}$ Universidad Regional Autónoma De Los Andes Uniandes-Ibarra. Facultad de Dirección de Empresas. Carrera de Empresas Turísticas y Hoteleras Tesis De Grado Previo A La Obtención Del Título De Ingeniera en Dirección Y Administración De Empresas Turísticas y Hoteleras.
} 
puede observar la presente tesis doctoral toma como referencia marcos de estudio de diferentes ámbitos. No obstante una de las clave de esta investigación es la identificación de las conexiones que se establecen entre la vida cotidiana, la identidad cultural y los valores patrimoniales con el fin de explicar la necesidad reconocer la funciones sociales del patrimonio en la actualidad.

\section{Métodos}

Si bien sirvieron de apoyo algunas herramientas de las ciencias técnicas, se tiene en cuenta la génesis histórica y la impronta antropológica de este fenómeno cultural y se aplica un enfoque integral que permita comprender la obra arquitectónica no solo desde su funcionalidad utilitaria, sino también desde su concepción estética e implicaciones en el orden económico y sociocultural, al ser la vivienda escenario de manifestaciones de la vida cotidiana; donde se dirimen temas esenciales de la economía familiar y comunitaria y se replican las tradiciones, los imaginarios colectivos, las manifestaciones artísticas como la música instrumental, el canto, la danza, la gastronomía, y otras expresiones de la identidad, dentro y asociadas a ella.

Esta investigación se ha desarrollado desde la perspectiva de la metodología cualitativa y del paradigma hermenéutico. Se trata de un estudio de caso por lo que fue fundamental la utilización del método de la observación científica -documental, monumental de campo y participante-; muchos de los datos obtenidos mediante la revisión de documentos fueron corroborados en el trabajo de campo. Los inmuebles constituyen el mejor testimonio y ofrecen informaciones de valor inestimable; así como, las vivencias del investigador a propósito de las manifestaciones del patrimonio intangible.

Para no fragmentar el análisis, se hará referencia al emplazamiento (ubicación del inmueble en el espacio), la planimetría (plantas, distribución espacial y relación funcional), soluciones volumétrico-formales (fachadas y cubiertas) y soluciones técnico-constructivas (pisos, técnicas, sistema y materiales).

El criterio de selección del periodo obedece a que el año 1960 marca un antes y un después en la historia de esta temática; debido a la promulgación y aplicación de la Ley de Reforma Agraria en 1964, con su consecuente impacto en el mejoramiento de la calidad de vida de los habitantes del Valle del Chota de esa época, uno de cuyos resultados fue precisamente el cambio en la concepción de la vivienda vernácula aun cuando continuó ligada al sistema agrícola y apegada a la tradición. Este marco temporal se cierra en 1990, fecha a partir de la cual, tiene lugar una tendencia a la construcción de viviendas basada en modelos urbanos y se evidencia por tanto, un paulatino abandono de las técnicas tradicionales.

En el año 2010, la Dirección Nacional de Patrimonio del Ecuador hizo un inventario de las viviendas consideradas como vernáculas. En esa oportunidad fueron censadas 20 casas por lo que se ha decidido trabajar con todo el universo dado lo exiguo del mismo. Para el desarrollo de la presente investigación se realizó una actualización e interpretación de ese inventario a partir de las variables definidas para el análisis de la tipología ${ }^{6}$.

\footnotetext{
${ }^{6}$ Cada vivienda tiene su ficha técnica. Por su importancia, al final del trabajo se anexa el inventario; este pudiera ser considerado un aporte práctico de la tesis.
} 


\section{Resultados}

De acuerdo con el censo realizado en el Valle del Chota en los años 1953, 1954 y 1955, ${ }^{7}$ las primeras construcciones de los negros que habitaron la zona pueden haber recibido varias influencias identificables en las edificaciones actuales: la vivienda de planta redonda de techo cónico - bohíos- pastenses, hechos de bahareque y adobe de tierra batida, de planta circular, con una sola puerta; la vivienda tipo imbabureño o kara, de planta rectangular con paredes delgadas, a veces de piedra de río, con recio empañete de barro preparado o de adobones superpuestos y techo a dos aguas con ligamentos de paja con su única puerta en la parte frontal y un amplio corredor sostenido por pilares de capulí, lechero o chahuarquero. Las características de la vivienda de los habitantes del Valle del Chota están determinadas por la altitud. En estos cálidos valles con cambios bruscos de temperatura, históricamente se ha preferido el tipo de construcciones ligeras idóneas para resistir el calor sofocante del día y el frío penetrante de la madrugada. Los factores que determinan la estructura de la vivienda del afro ecuatoriano son de orden geográfico, pero también se suman los de índole cultural. Los hábitos y costumbres arraigados son evidentes tanto en las características de las edificaciones como en su disposición en el espacio.

Como resultado, se determinaron las pautas de asentamiento de una comunidad cuya génesis está marcada por la presencia indígena y negra en un territorio otrora propiedad de los Jesuitas, enclavada en un sitio matizado por las peculiaridades de la geografía andina.

Las viviendas vernáculas que han logrado sobrevivir en el territorio son en su mayoría de un solo nivel. En el Valle del Chota predomina un tipo de vivienda que pudiera definirse como de planta rectangular, más ancha que profunda, con dos crujías y tres espacios en cada una de ellas, no presentan baño, la fachada principal es la única que se destaca por la presencia de vanos y su disposición simétrica, no presenta corredor y la cubierta es a cuatro vertientes con tejas de barro en su terminación cuya estructura interna es visible dada la ausencia de falso techo. Los materiales usados son la piedra para los cimientos, el adobe para las paredes, el cemento para los pisos y el barro para las cubiertas.

De la vivienda devenida en antecedente de esta se conserva, por tanto, la planimetría rectangular y la cubierta a cuatro vertientes; sin embargo, la primera difiere en que ya no será un espacio único sino que existe más de una crujía, subdividida para la satisfacción de necesidades de naturaleza diversa. La cubierta, aunque tiene el mismo número de vertientes cuenta con un material relativamente nuevo en su terminación: las tejas de barro cocido. El bahareque es desplazado por el adobe como técnica predominante en la concepción de paredes y el piso de tierra es sustituido por el de cemento. Finalmente, el corredor en la fachada principal prácticamente desaparece.

La belleza de estas construcciones radica en lo auténtico del material; no aparece ningún elemento incorporado para que cumpla esa función. Es el color de la tierra cocida, tanto en los muros como en los techos con tejas de barro en su terminación, en contraste con el color de las

\footnotetext{
${ }^{7}$ Este censo es realizado por el, ya mencionado, Instituto Ecuatoriano de Antropología y Geografía durante una investigación en todo el Valle del Chota y Salinas. En el informe que rinden, una de las comunidades más referidas es Chalguayacu, lo cual resulta de gran importancia para esta investigación.
} 
piedras de los cimientos, lo que las dota de esa hermosura que solo tienen las cosas simples; esas que están ahí desde hace mucho tiempo, sin que se hubiese aprendido a apreciarlas y, por alguna razón, no es posible desprenderse de ellas.

En la elaboración de estas construcciones fue esencial la existencia de los materiales necesarios tomados de la naturaleza; madera, tierra, caña y paja eran abundantes en el extenso valle; las piedras eran recogidas en las márgenes del río Chota. No había que trasladarse a otro sitio para buscar los recursos, todo estaba allí, al alcance de la mano, producto del generoso medio ambiente; de ahí la relación que se establece entre las construcciones y su entorno ${ }^{8}$. Los diseñadores y ejecutores de obras eran los propios miembros de la comunidad.

Esta es, por tanto, un tipo de arquitectura económica y que se adecua al contexto "involucra elementos culturales, arquitectónicos, técnicos y ambientales en la óptima solución de sus viviendas" (Saravino, 2011, s.p). Las relaciones forjadas entre los elementos están entre los referentes para el examen de sus fundamentos en la concepción y perfeccionamiento de una arquitectura contemporánea.

Sin embargo, llama la atención que en las comunidades Chota y Juncal quedan muy pocos ejemplos de esta tipología y están en condiciones de deterioro realmente preocupantes. ${ }^{9}$ En Chalguayacu, aunque se conserva un mayor número de inmuebles, dentro de poco la situación será similar, pues no existe, según se ha podido indagar, ningún proyecto con vistas a la conservación y rescate de esta arquitectura.

Finalmente, la preservación de estos caseríos significa salvar el espacio donde, aún hoy, sobreviven algunas tradiciones de los afro ecuatorianos que habitan estos lares, dicho de otro modo, la arquitectura vernácula y el espacio en la que ella se encuentra emplazada, es el marco en el que estas se despliegan; constituyen el sostén físico de múltiples expresiones culturales, por lo que la conservación de esta parte del patrimonio tangible implica, por extensión, la supervivencia de manifestaciones del patrimonio intangible en su verdadero contexto. La comunidad es el espacio idóneo para el desarrollo y supervivencia de sus tradiciones más genuinas.

\section{Discusión}

En Ecuador, las zonas rurales han sido tradicionalmente relegadas por las políticas públicas, por tanto, cualquier medida que se tome para visualizar esta situación es ya meritoria. ${ }^{10}$ Dada la complejidad de la problemática campesina-rural caracterizada por la dispersión de los asentamientos, pobreza endémica, degradación ambiental, alta migración, baja productividad,

\footnotetext{
${ }^{8}$ Las tejas se fabricaban en hornos emplazados en sitios no muy cercanos desde donde eran trasladadas hasta el caserío.

${ }^{9}$ En la comunidad Chota se encontró a una familia en pleno proceso de recogida de escombros porque dos inmuebles habían sufrido derrumbe parcial como consecuencia de la inclemente lluvia de la noche anterior. En el valle, existen numerosas viviendas deshabitadas y abandonadas que se deterioran progresivamente hasta la destrucción total.

${ }^{10}$ Se ha dado a conocer que la actual administración gubernamental ha tomado medidas en el sentido de homologar el bono para vivienda rural con el bono para vivienda urbano-marginal.
} 
entre lo más relevante; enfrentar el tema de la vivienda demanda asumir posturas más integrales ${ }^{11}$ (Pinto \& Ruiz, 2009).

De acuerdo con (Arrata, 2014) la vivienda vernácula fue común en la serranía ecuatoriana hasta avanzado el siglo XX, década de 1960 aproximadamente, fecha a partir de la cual es reemplazada, progresivamente, por el uso de materiales de construcción y diseños no compatibles y ajenos al entorno. Llama la atención, en cambio, que las casas inventariadas en Chalguayacu fueron construidas después de 1960, lo cual pudiera indicar la supervivencia de una tradición más allá del marco temporal señalado; por otra parte, reafirma el criterio de que "estos tipos de edificación pueden tener una temporalidad distinta, pueden tener un origen histórico o contemporáneo" (Aguillón, Benítez y Arista, s.f, s.p).

"A diferencia de las arquitecturas históricas o de estilo, la arquitectura tradicional no presenta una evolutiva general concordante con los cambios de soportes económicos, tecnológicos y culturales de la vida urbana, se encuentran detenidas en la etapa que las concibió" (Maldonado y Vela, citado por Tillería 2010, p. 14). Estas viviendas, responden a peculiaridades que marcan la evolución de la comunidad y plantea el modo intemporal de construir, "se trata de un proceso a través del cual el orden... surge de la naturaleza interna de la gente... El campesino... no pierde tiempo diseñando su casa. Piensa... dónde y cómo levantarla, y luego comprende su construcción” (Christopher Alexander, citado por Tillería 2010, p. 14).

De acuerdo con la bibliografía consultada, en Ecuador, tal y como sucede en otros sitios, este tipo de edificaciones ha evolucionado a lo largo del tiempo. ${ }^{12}$ La vivienda campesina -para algunos, simplemente, "la choza"- originalmente con techo de paja, incorpora luego otros materiales como las tejas de barro para las cubiertas, sin embargo, desde el punto de vista espacial, durante mucho tiempo persistió el concepto de habitación única, en la cual debían ser satisfechas varias necesidades, desde descansar, elaborar alimentos, comer y hasta dormir.

Este modo de construir no responde a conceptos pre-establecidos de diseño, es el resultado de un largo proceso de aprendizaje y experimentación que, a través del tiempo, los miembros de un mismo grupo desarrollan; de ahí la importancia de la calidad de la relación y comunicación entre ellos y con el entorno. Por extensión, la forma, función y espacio se relacionan con las actividades del grupo no son resultado de la creación individual sino respuesta a las necesidades, posibilidades tecnológicas y recursos materiales, o sea, dependen de la realidad, del tiempo y estilo de vida (Saravino, 2011).

La vivienda vernácula es resultado del largo proceso evolutivo de un procedimiento pragmático demostrado por generaciones y que, constituye, la opción más funcional a múltiples actividades, tanto de índole económica en el ámbito rural, como socio culturales propias de las relaciones tradicionales, por lo tanto, en estos espacios existe un valor útil (Torres, s.f). En Chalguayacu las construcciones de bahareque y paja fueron tan funcionales, en su momento, como lo serían luego las de adobe y tejas de barro. La casa fue, desde siempre, un espacio dotado

\footnotetext{
${ }^{11}$ Vanessa Pinto y Silvana Ruiz sugieren enfocar la problemática desde la interculturalidad y la sustentabilidad social, económica y ambiental.

${ }^{12}$ En Cuba, por ejemplo, tiene lugar una evolución en la casa campesina que va dejando atrás su forma original para convertirse en la casa de madera.
} 
del ajuar doméstico preciso para la satisfacción de necesidades elementales y ámbito para el disfrute de expresiones que enriquecen el espíritu de sus moradores.

Se considera que en la actualidad, las técnicas constructivas vernáculas forman parte del repertorio de alternativas disponibles para enfrentar los problemas ambientales. En la práctica cotidiana, los constructores tradicionales conocen, a menudo mejor que los propios arquitectos, cómo adaptarse a condiciones cambiantes. Esto se debe, entre otras razones, a que ellos pueden asumir el riesgo de experimentar opciones que ofrecen resultados inciertos, sin temor a perder prestigio profesional y contratos de trabajo. Estos artífices sin escuela tienen un especial talento para ubicar sus edificios en el medio natural; se adaptan al entorno pues tienen un agudo sentido para manejar problemas prácticos, de ahí que sus propuestas sean eternamente válidas, así como, las herramientas que utilizan para construirlas (Fernández, 1991, s.p). Son, además, muy diversas; razón por la cual no es posible definir tipos, "ella no ocupa un catálogo de partes que puedan ser combinadas, cada sistema resultante es único e irrepetible” (Tillería, 2010, p.14)

A propósito de la situación que afronta actualmente la arquitectura vernácula en el Valle del Chota, habría que tener en cuenta que el vínculo o intercambio con la ciudad a raíz de los procesos migratorios condicionados, entre otras razones, por ese fenómeno en que se ha convertido el football, ${ }^{13}$ ha producido cambios en el modo de vida y de pensar de muchas familias y ha impuesto, casi de manera inconsciente nuevos paradigmas, dada la necesidad de exteriorizar una solvencia económica y un ascenso social reflejado, en primera instancia, en la vivienda.

Las casas construidas con tierra están expuestas a deteriorarse con mayor rapidez, pues su resistencia a factores como la lluvia y la humedad que caracterizan la región andina, es menor que la de obras más sólidas y, para llevar a cabo el mantenimiento de las mismas, no basta el interés de los moradores, evidente en el trabajo de campo, sino que es preciso tener los recursos que lo permitan. Hay una relativa escasez de algunos materiales y la carencia de mano de obra conocedora de técnicas que han ido quedando en el olvido.

Estos resultan emplazamientos relativamente dispersos en medio de la serranía y, más de un especialista se ha preguntado si merecen la atención de los poderes públicos, en especial, de las entidades encargadas de la protección del patrimonio cultural y natural. ${ }^{14}$

En realidad, las acciones estatales encaminadas a la protección del patrimonio construido de la región andina, específicamente de la arquitectura vernácula, a través de proyectos de conservación y restauración, no han sido sistemáticos ni suficientes; tampoco existe una política que estimule el mantenimiento de lo que ha sobrevivido y, mucho menos, la calificación de una

\footnotetext{
${ }^{13}$ Con la clasificación de Ecuador para los mundiales de football Corea-Japón (2002) y Alemania (2006), los afrochoteños alcanzan una visibilidad insospechada. El football ha contribuido a la construcción de un nuevo imaginario de identidad en el valle del Chota, cercano a los modelos que publicita el mundo de los ricos y famosos, los futbolistas del valle del Chota son propuestos como un nuevo modelo social en el cual reconocerse y representarse (Ortiz, 2011).

${ }^{14}$ Arrata (2014) hace un profundo análisis sobre este particular. También (Cruz, 2015), entre otros autores.
} 
mano de obra que pueda intervenir en estas construcciones. ${ }^{15}$ En las nuevas edificaciones no se utilizan técnicas ni materiales tradicionales, a pesar de las ventajas que ello tendría pues permitiría su favorable inserción en el medio y pudiera ser un gran reto a la imaginación creadora de los proyectistas.

La arquitectura vernácula de Chalguayacu está directamente asociada a toda una serie de tradiciones que se conservan en la comunidad. El modo de vida, los hábitos y costumbres arraigados y heredados imponen determinados matices en la concepción del hábitat y, al mismo tiempo, es en este contexto donde manifestaciones como la música, la danza, el vestuario, la alimentación, entre otras, encuentran el espacio ideal para su despliegue. Esta es una muestra de la estrecha relación que se establece entre lo tangible y lo intangible, en cuyo centro está el hombre. De modo que, cuando se hace referencia a la conservación se trata de la defensa, no de una manifestación en particular, sino de un todo patrimonial constituido por bienes y expresiones de un valor incalculable.

Se trata de una tipología arquitectónica económica; aprovecha los recursos que la naturaleza ofrece, se adapta al entorno, no atenta contra la ecología, se inserta de manera armónica al paisaje por lo que deviene vía idónea en la búsqueda de soluciones sustentables para la arquitectura contemporánea; sin embargo, la inexistencia de una política adecuada, la carencia de mano de obra especializada y de algunos materiales necesarios atentan contra su conservación y hoy amenaza con desaparecer. Es preciso encontrar vías que permitan su salvaguarda, lo cual es interés de muchos moradores que permanecen en la comunidad y desean conservar sus viejas viviendas a pesar de las nuevas construcciones que se levantan, ajenas a esa tradición.

\section{Conclusión}

A partir de 1960 la vivienda vernácula del Valle del Chota supera la que había distinguido a todo el valle durante años; se asume una tipología en la existe un mayor número de espacios con funciones bien definidas, cimientos de piedra, paredes de adobe -a veces enlucidascubierta de tejas a cuatro vertientes y piso de cemento. La concepción de un inmueble en la que se incorpora lo estético, aun cuando no supere lo funcional, con un emplazamiento en el espacio que delata cierto orden, también habla de un cambio a nivel de mentalidad de individuos que están más arraigados y cuyas condiciones económicas han mejorado.

La conservación y rescate de la arquitectura vernácula responde, entre otras razones, a la necesidad de salvaguardar el patrimonio construido. De la relación que se establece entre la tipología caracterizada en el primer epígrafe de este capítulo y el entorno, se deriva una unidad de gran valor estético que contribuye al enriquecimiento del paisaje rural; a ello habría que sumarle las manifestaciones del patrimonio intangible que se han desarrollado históricamente en ese contexto, cuya preservación también se vería amenazada.

Esta vivienda deja atrás el bahareque y la paja, técnicas constructivas heredadas de los indígenas para asumir el adobe, en un momento en el que en otros espacios se aboga por la

\footnotetext{
${ }^{15}$ En Cuba se ha puesto en práctica la creación de escuelas-taller para la formación de personal especializado en oficios que se han ido perdiendo, por ejemplo, la "Gaspar Melchor" de Jovellanos, y la "Hugo Luisi" en Santiago de Cuba. Se trata de experiencias aplicables a otros contextos.
} 
última tecnología pero no es lícito violentar la evolución en un ámbito que difiere del urbano y que, por demás, ha estado plagado por la pobreza. El ritmo de desarrollo de una comunidad como esta, no puede ser el mismo ni tiene por qué renunciar a lo que le es inherente. Se ha demostrado que la búsqueda de soluciones ajenas al contexto, a largo plazo fracasa; es preciso tener en cuenta las características locales y, en eso, los antecesores fueron sabios. Si actualmente los investigadores en el campo de la construcción están haciendo propuestas a partir de la arquitectura vernácula es porque se ha demostrado que es sustentable e idónea para estos tiempos.

\section{Agradecimientos}

A los habitantes del Valle del Chota que han colaborado con esta investigación.

\section{Bibliografía}

Antón, J. (2010). La experiencia afrodescendiente y la visibilidad estadística en el Ecuador.

Antón, J. \& Tuaza, L. (s.f). Salvaguardia del patrimonio cultural inmaterial de los afrodescendientes en América Latina. Informe sobre la situación del PCI afrodescendiente en Ecuador. Auspiciado por la UNESCO.

Arrata, M. (2014). Destrucción de la arquitectura vernácula campesina en los andes ecuatorianos. Agusvinnus, 0(9). ISSN 2362-6526.

Coronel, R. (1991). El valle sangriento: de los indígenas de la coca y el algodón a la hacienda cañera jesuita, 1580-1700. (Vol. 4). Quito: Ediciones Abya Yala.

Chalá, J. (2006): Chota profundo. Antropología de los afrochoteños. Quito: Abya-Yala.

ICOMOS. (1999). Carta del Patrimonio Vernáculo Construido, XII Asamblea General del ICOMOS, México.

López, Francisco. (1987). Arquitectura vernácula en México. México: Editorial Trillas.

Naranjo, M. (2016a). Aportes de la herencia cultural y espiritual de los pueblos afroecuatorianos a la construcción del buen vivir. UTN-Ibarra-Ecuador. ISBN 9789942-8631-2-6.

Nurnberg, D (1882). Arquitectura vernácula en el litoral ,Guayaquil, Banco Central del Ecuador.

Oliver, P. (1997). Encyclopedia of Vernacular Architecture of the World, Vol. III. Cultures and Inhabitants, Cambridge University Press, United Kingdom.

Ortiz, Enrique(1999). Arquitectura vernácula - Arquitectura con arquitectos: una interacción fecunda en riesgo de extinción (Cultura urbana). En: Revista Ciudad Alternativa. Pensar en las ciudades. Quito: CIUDAD, (no. 14, III Época, Número especial, 1998-99): pp. 141147. 
Padilla Pastrana, L. G. (2014). Desarrollo local y afrodescendencia: El caso de la parroquia de Salinas, en el Valle del Chota. (Trabajo de grado). FLACSO. Quito, Ecuador.

Pabón, I. (2006). Procesos de construcción identitaria en las comunidades negras de la cuenca Chota-Mira en tres generaciones: abuelos, adultos mayores y jóvenes (Trabajo de grado). Universidad Andina Simón Bolívar. Quito, Ecuador.

Padilla Pastrana, L. G. (2014). Desarrollo local y afrodescendencia: El caso de la parroquia de Salinas, en el Valle del Chota. (Trabajo de grado). FLACSO. Quito, Ecuador.

Peñaherrera, P., \& Costales, A. (1959). Coangue o Historia Cultural y Social de los negros del Chota y Salinas. Investigación y elaboración. Instituto Ecuatoriano de Antropología y Geografía. Ecuador, Quito.

Pinto, V, \& Ruiz, S. (2009). La vivienda rural en el Ecuador: desafíos para procesos sustentables e incluyentes sistematización: contrato social por la vivienda. Ecuador, Quito.

Saravino, M. (2011). Arquitectura indígena: fundamentos para la generación de una arquitectura contemporánea. [Recuperado el 07 de octubre de 2016 de http://repositorio.usfq.edu.ec/bitstream/23000/825/2/99846\%20(Tesis).pdf] Cataluña.

Tillería, J. (2010). La arquitectura sin arquitectos, algunas reflexiones sobre arquitectura vernácula. Revista AUS (8), pp.12 -15.

Yépez, D. (2012). Análisis de la arquitectura vernácula del Ecuador: propuestas de una arquitectura contemporánea sustentable. Universidad Politécnica de Cataluña. 